\title{
O Turnover dos profissionais de saúde na Estratégia Saúde da Família do Município
}

\section{de Rio Grande do Piauí-PI}

The turnover of health professionals in the Family Health Strategy in the City of Rio Grande do Piauí-PI

La rotación de profesionales de la salud en la Estrategia Salud de la Familia en la Ciudad de Rio Grande do

Piauí-PI

Recebido: 06/04/2021 | Revisado: 12/04/2021 | Aceito: 23/04/2021 | Publicado: 08/05/2021

\author{
Marceane Barros Lourenço \\ ORCID: https://orcid.org/0000-0003-2364-248X \\ Universidade Federal do Piauí, Brasil \\ E-mail: ceane_barros@hotmail.com \\ Kairlane Soares da Silva \\ ORCID: https://orcid.org/0000-0002-8557-3806 \\ Universidade Federal do Piaú, Brasil \\ E-mail: kairlanesoares10@gmail.com \\ Flávia Lorenne Sampaio Barbosa \\ ORCID: https://orcid.org/0000-0002-4804-9538 \\ Universidade Federal do Piauí, Brasil \\ E-mail: flsbarbosa@ufpi.edu.br
}

\begin{abstract}
Resumo
Este estudo analisa o turnover dos profissionais de saúde da Estratégia Saúde da Família do município Rio Grande do Piauí. Busca verificar a influência desse fenômeno nos integrantes das equipes e na qualidade dos serviços de saúde prestados à população. Trata-se de um estudo com abordagem qualitativa, em que foi aplicada entrevista com os sujeitos da pesquisa e analisado o índice e a média anual de turnover, além dos tipos de vínculos das contratações da ESF do município. Os resultados demonstram uma taxa de rotatividade instável, sendo alta em determinados períodos, além disso, a instabilidade também predomina na forma de contratação dos profissionais, sendo $75 \%$ por prazo determinado. A análise das entrevistas indica como principais causas da rotatividade a insatisfação desses profissionais, devido ao estilo de gestão autoritário, às condições de trabalho precárias, problemas de estrutura, sobrecarga de trabalho, ausência de leis trabalhistas que tragam segurança ao servidor e, sendo o principal problema, o fato de profissionais residirem em outros municípios. Esse contexto demonstra a necessidade de buscar melhorias em relação à liderança, às condições de trabalho, ao modo de gerir os recursos públicos, para que as práticas da ESF sejam realizadas na sua integralidade.
\end{abstract}

Palavras-chave: Rotatividade; Estratégia saúde da família; Satisfação no trabalho; Saúde pública.

\begin{abstract}
This study analyzes the turnover of health professionals in the Family Health Strategy in the city of Rio Grande do Piauí. It seeks to verify the influence of this phenomenon on the team members and on the quality of health services provided to the population. This is a study with a qualitative approach, in which an interview with the research subjects was applied and the annual turnover index and average was analyzed, in addition to the types of bonds in the municipality's ESF contracts. The results show an unstable turnover rate, being high in certain periods, in addition, instability also predominates in the form of hiring professionals, with $75 \%$ for a fixed period. The analysis of the interviews indicates that the dissatisfaction of these professionals is the main cause of turnover, due to the authoritarian management style, poor working conditions, structural problems, work overload, absence of labor laws that bring security to the server and, being the main problem, the fact that professionals live in other municipalities. This context demonstrates the need to seek improvements in relation to leadership, working conditions, the way of managing public resources, so that the practices of the ESF are carried out in their entirety.
\end{abstract}

Keywords: Turnover; Family health strategy; Job satisfaction; Public health.

\section{Resumen}

Este estudio analiza la rotación de profesionales de la salud en la Estrategia Salud de la Familia en la ciudad de Rio Grande do Piauí. Se busca verificar la influencia de este fenómeno en los integrantes del equipo y en la calidad de los servicios de salud brindados a la población. Se trata de un estudio con enfoque cualitativo, en el que se aplicó una entrevista a los sujetos de investigación y se analizó el índice de rotación anual y la media, además de los tipos de contratos laborales en el FSE del municipio. Los resultados muestran una tasa de rotación inestable, siendo alta en determinados períodos, además, la inestabilidad también predomina en la forma de contratación de profesionales, con 
un 75\% por un período fijo. El análisis de las entrevistas indica que el descontento de estos profesionales es la principal causa de la rotación, debido al estilo de gestión autoritario, las precarias condiciones laborales, problemas estructurales, sobrecarga de trabajo, ausencia de leyes laborales que traigan seguridad al servidor y, siendo el principal problema el hecho de que los profesionales vivan en otros municipios. Este contexto demuestra la necesidad de buscar mejoras en relación al liderazgo, las condiciones laborales, la forma de gestionar los recursos públicos, para que las prácticas de la ESF se lleven a cabo en su totalidad.

Palabras clave: Facturación; Estrategia de salud de la família; Satisfacción laboral; Salud pública.

\section{Introdução}

O turnover (rotatividade) é caracterizado por ser a relação entre admissões e demissões de funcionários em uma empresa e acontece devido a vários fatores, sendo tema de pesquisas pelas quais se busca descobrir soluções que reduzam essa movimentação de colaboradores, pois formar uma equipe competente é uma tarefa difícil, devido à alta competitividade e a carência de pessoas qualificadas no mercado de trabalho como mostra a pesquisa da Korn Ferry (The Global Talent, 2019).

Sendo assim, perder um colaborador acarreta prejuízos muito além do financeiro, pois ao se desligar essa pessoa leva consigo toda uma bagagem de conhecimentos e experiências úteis àquela empresa. E, quando alguém novo é contratado para a vaga, há de se investir em todo um processo de adaptação e capacitação, gerando atrasos e custos (Mota, Levy \& Coelho, 2016).

Estudos mostram que nas organizações públicas o turnover pode aumentar os custos com pessoal, além de prejudicar a eficiência, diminuir a produtividade e o desempenho das equipes, trazer sobrecarga de atividades aos demais servidores e impactos na motivação. Entende-se que a troca de servidores pode ocasionar na redução da experiência profissional em determinados assuntos (Pereira \& Almeida, 2017).

E, em relação aos servidores da saúde, essa redução da experiência requer atenção especial, conforme estudo de Tonelli, et al. (2018), a experiência desse profissional é adquirida com a vivência no território e no desenvolvimento das práticas em saúde responsável pela "troca de saberes entre os trabalhadores da saúde e os usuários" (Santos \& Miranda, 2016, p. 6), com isso, constata-se que o afastamento do profissional de saúde pode acarretar prejuízo ao serviço prestado à população.

A presente pesquisa norteou esse tema para o setor público, especificamente, para o serviço de saúde, por meio da Estratégia Saúde da Família (ESF) do município de Rio Grande do Piauí, com o intuito de se estudar a movimentação dos servidores das equipes e a possível quebra do vínculo gerado entre esses profissionais e a população, na medida que a proposta da saúde da família da Política Nacional da Atenção Básica (PNAB) (2012) fundamenta-se neste vínculo.

Diante do exposto, elaborou-se o seguinte questionamento: como o turnover dos profissionais da Estratégia Saúde da Família interfere no vínculo gerado entre esses profissionais e a população? Tendo como objetivo geral, analisar de que forma o turnover dos profissionais da Estratégia Saúde da Família interfere no vínculo entre esses profissionais e a população do município de Rio Grande do Piaú́.

Além disso, atingir os seguintes objetivos específicos: identificar o índice de turnover; descobrir quais os fatores que influenciaram o desligamento dos profissionais das equipes ESF do município; e por fim buscar alternativas para a fixação desses profissionais, por meio das descobertas de autores e sugestões dos sujeitos da pesquisa.

Acredita-se que o estudo da rotatividade dos profissionais da ESF se mostra importante para a gestão da saúde municipal, para a sociedade e para a ciência. Contribui com a gestão municipal na busca da economicidade e da eficiência do serviço público de saúde, em razão de estudos apontarem a rotatividade como sendo um dos maiores problemas para as organizações, pois gera custos na oferta de treinamentos aos profissionais e custos com a reposição de pessoal (Campos \& Malik, 2008). É importante para a sociedade, pois visa contribuir para o aprimoramento dos serviços de saúde, no alcance de benefícios, como por exemplo, melhoria no cuidado individual e coletivo e integralidade do atendimento (PNAB, 2012). Se torna importante também para a ciência visto que, diante do estudo bibliográfico realizado, foi notória a quantidade resumida 
de estudos voltados para o tema da rotatividade de profissionais na ESF.

\section{Fundamentação Teórica}

\subsection{Turnover na Gestão Pública}

O contrato de trabalho na gestão pública se difere do privado em dois pontos: primeiro na forma de admissão, que ocorre por meio do concurso público e segundo na demissão, que deve seguir a legislação trabalhista adotada, esta podendo ser estatutário (Lei 8.112/90) ou celetista (Consolidação das Leis do Trabalho) o que garante maior estabilidade aos vínculos empregatícios. Dentre esses, o contrato celetista é o menos estável, por ser mais flexível em suas regras de desligamento (Departamento Intersindical de Estatística e Estudos Socioeconômicos [DIEESE], 2016).

Segundo estudo do DIEESE realizado em 2016, o turnover da gestão pública, no segmento celetista, subiu de 14,5\% em 2003 para 50,1\% em 2014, excluindo os desligamentos por falecimento, aposentadoria, transferência e demissão a pedido.

Esse aumento demonstra a importância de se investigar as causas do turnover na gestão pública, no intuito de buscar a redução dos custos e aumentar a eficiência dos serviços prestados à população, como é o caso dos serviços de saúde do modelo proposto pelo PNAB, em que a troca de pessoal pode causar prejuízos ao interromper o vínculo entre o profissional da Estratégia Saúde da Família e a comunidade (Medeiros, et al., 2010).

\subsection{Estrategia Saúde da Família}

Inicialmente, o modelo de saúde da Atenção Básica implantado no Brasil pelo Ministério da Saúde, em 1994, foi chamado de Programa Saúde da Família (PSF), apresentando-se como um novo modelo de atenção à saúde que fosse voltado para a prevenção de doenças e proteção da saúde com atenção às pessoas; diferente do modelo anterior que era voltado para práticas curativas e hospitalares, fato que aumentava bastante os custos com tratamentos e medicamentos.

Conforme Campos (2005), no modelo do PSF, o foco da atenção se dá sobre a família e a comunidade, sendo fortemente valorizado o vínculo entre os profissionais das equipes e a população. As equipes foram implantadas em todas as unidades de saúde do território nacional nos anos 90, e eram inicialmente compostas por médicos, enfermeiros, dentistas, auxiliares e técnicos de saúde, responsáveis pela promoção da saúde da população.

A expansão da Atenção Básica em todo o território nacional ocorreu em 2006, por meio da portaria n. 648/GM/MS, na qual ocorreu o Pacto de Gestão entre o Ministério da Saúde e os municípios, distrito federal e as secretarias estaduais e municipais de saúde. Com o sucesso do programa para a Atenção Básica no Brasil, o PSF se consolidou como estratégia prioritária do Ministério da Saúde e passou a se chamar Estratégia Saúde da Família (ESF).

Em janeiro de 2008, a portaria n. 154 GM/MS criou o Núcleo de Apoio à Saúde da Família (NASF) para a ampliação da capacidade de resposta das equipes aos problemas da população, com isso ampliou o atendimento da ESF com novas especialidades e novos profissionais como nutricionistas, fisioterapeutas, fonoaudiólogos, entre outros, atuando de forma integrada em ações compartilhadas com os profissionais das equipes do ESF. Com a inclusão desses profissionais, espera-se a ampliação da atenção e cuidado, melhorando assim a qualidade de vida das pessoas (PNAB, 2012).

\subsection{Turnover na Estrategia Saúde da Família}

As equipes da ESF são capacitadas para terem contato com a realidade das famílias que atendem, de modo a tornarem-se sensíveis às suas necessidades, com isso surge o vínculo que é um facilitador para o cuidado da saúde da comunidade (Borges, Santos \& Fischer, 2019). O índice de turnover dos profissionais dessas equipes, se considerado alto, 
pode vir a prejudicar a efetividade deste vínculo, e isso pode acarretar problemas para o desenvolvimento da ESF no município (Medeiros, et al., 2010).

Estudos mostram que é de fundamental importância que os profissionais de saúde permaneçam nas equipes, pois isso é tido como um dos fatores críticos para o sucesso da ESF (Tonelli, et al., 2018). Portanto, analisar os índices de rotatividade desses profissionais é uma estratégia importante para a continuidade dos trabalhos das equipes da ESF. Porém, essa análise não parece ser uma tarefa fácil, visto que Borges, Santos e Fischer $(2019$, p. 6) afirmam que "resolver esse problema constitui-se um desafio, tendo em vista a multiplicidade das causas", dentre elas; contratos inseguros, (Borges, Santos \& Fischer, 2019; Campos \& Malik, 2008) salários defasados, (Borges, Santos \& Fischer, 2019; Magnago \& Pierantoni, 2015) condições precárias de trabalho, (Borges, Santos \& Fischer, 2019; Campos \& Malik, 2008; Magnago \& Pierantoni, 2015) falta de equipamentos e materiais, além da insatisfação com o trabalho, ( Borges, Santos \& Fischer, 2019; Tonelli, et al., 2018).

Dentre os fatores geradores de rotatividade nas equipes da ESF, os autores citam como a principal e mais importante a insatisfação com o trabalho, fator este, que influencia a permanência ou a saída do trabalho (Borges, Santos, \& Fischer, 2019; Campos \& Malik, 2008). Para Tonelli, et al. (2018, p. 4) "a rotatividade não é a causa, mas a consequência, de fenômenos internos e externos à organização", portanto, percebe-se que uma possível solução para a permanência dos profissionais está na busca da satisfação destes com o trabalho.

Posto disso, o turnover pode ser positivo quando pessoas ineficientes deixam a organização ou negativo, quando há perda de pessoas qualificadas, prejudicando, assim, o desenvolvimento organizacional (Tonelli, et al., 2018). Dessa feita, no âmbito da ESF, considerando a importância do conhecimento entre o profissional de saúde e o paciente, ou seja, do vínculo construído entre as equipes e a população, o efeito negativo dessa rotatividade não garantirá a continuidade das ações de saúde por meio da relação de confiança, aumentando, assim, os riscos no tratamento pelo desconhecimento da história de vida do paciente (PNAB, 2012).

Outro aspecto negativo do turnover é o financeiro, visto que, quando ocorre a troca de servidores, há de se investir novamente em treinamentos (Medeiros, et al., 2008). Pela normatização do PNAB (2012) dentre as atribuições específicas das equipes do ESF, consta a participação em treinamentos e capacitações.

\section{Metodologia}

O estudo classificou-se qualitativamente, de acordo com Minayo (2001), a pesquisa qualitativa trabalha com o universo de significados, motivos, aspirações, crenças, valores e atitudes, o que corresponde a um espaço mais profundo das relações, e descritivamente, que segundo Gil (2002), esse tipo de pesquisa, serve a princípio para descrever as características de determinada população ou fenômeno ou ainda para estabelecer relações entre as variáveis, com pesquisa bibliográfica sendo realizada pela busca de publicações no sites da base de dados da SPELL (spell.org.br), SCIELO (scielo.org) e Google Acadêmico (scholar.google.com.br).

O locus de pesquisa foi o município de Rio Grande do Piauí-PI, que está localizado a 388 km da capital Teresina, com uma população de 6.273 pessoas (Instituto Brasileiro de Geografia e Estatística [IBGE], 2010). Atualmente, possui 3 (três) equipes do ESF e 1 (uma) equipe do Núcleo Ampliado de Saúde da Família (NASF) implantadas. E, os sujeitos da pesquisa compreenderam 8 (oito) pessoas conforme mostra o Quadro 1. 
Quadro 1- Descrição dos entrevistados.

\begin{tabular}{|l|l|l|}
\hline \multicolumn{1}{|c|}{ Função } & \multicolumn{1}{|c|}{ Sujeitos } & \multicolumn{1}{c|}{ Quantidade } \\
\hline Gestor & Secretário Municipal de Saúde & 01 pessoa \\
\hline \multirow{2}{*}{ Servidores ativos } & $\begin{array}{l}\text {-Enfermeiro da Estratégia Saúde da Família } \\
\text {-Dentista do Programa de Saúde Bucal }\end{array}$ & \multirow{2}{*}{02 pessoas } \\
\hline Servidores desligados & Enfermeiras da Estratégia Saúde da Família & \multirow{2}{*}{02 pessoas } \\
\hline \multirow{2}{*}{ Comunidade (usuários) } & Usuário da àrea de atendimento da equipe I & \multirow{2}{*}{03 pessoas } \\
\cline { 2 - 3 } & Usuário da àrea de atendimento da equipe II & \\
\cline { 2 - 3 } & Usuário da àrea de atendimento da equipe III & \\
\cline { 2 - 3 } & &
\end{tabular}

Fonte: Elaborado pelas autoras, com base na pesquisa realizada.

Como procedimento de coleta de dados foram realizadas entrevistas, previamente, consentidas (assinados os termos de livre consentimento esclarecido), respeitando, por certo, os objetivos do estudo. Ademais, a confidencialidade foi garantida aos sujeitos entrevistados, sendo representados pelos símbolos: E.01, S.01, S.02, D.01, D.02, C.01, C.02 e C.03.

Para tanto, foi elaborado um roteiro de entrevista contendo 12 questões abertas e subjetivas. Todas as entrevistas foram gravadas entre os dias 14/01 a 20/01/2021 e, literalmente, transcritas para a análise dos resultados.

Complementando as fontes de evidências do estudo, recorreu-se a pesquisa documental, por meio dos dados coletados nos registros do setor de gestão de pessoas da Secretaria Municipal de Saúde, bem como pelos registros dos profissionais nos sistemas do Ministério da Saúde; Cadastro Nacional de Estabelecimentos de Saúde (CNES) e Sistema de informação da Atenção Básica (SIAB). A finalidade é a obtenção da taxa de turnover que foi calculada através da planilha Ferramenta Indicador de Turnover desenvolvida pela coaching Vanusa Cardoso (material.vanusacardoso.com.br) realizado o cálculo por ano, com dados do período de 2016 a 2020, entre os profissionais em exercício e os que já se desligaram do município.

A análise dos dados foi feita de forma qualitativa. Para a análise das entrevistas foi utilizado o método de análise de conteúdo, (Bardin, 2004) na qual trechos das entrevistas foram recortadas em redor de cada categoria elencada. E, para os dados secundários, aplicou-se estatística descritiva, por meio do cálculo do índice de turnover e das porcentagens apresentadas dos dados.

Por meio da análise dos resultados, destacaram-se 5 (cinco) categorias que foram direcionadas pelo discurso dos entrevistados, conforme o Quadro 2.

Quadro 2 - Categoria e Base Teórica de Pesquisa.

\begin{tabular}{|l|l|l|}
\hline \multicolumn{1}{|c|}{ Categoria } & \multicolumn{1}{|c|}{ Teóricos } & \multicolumn{1}{c|}{ Público associado } \\
\hline Papel do gestor & (Medeiros, et al., 2010, p. 5) & Gestor municipal da saúde \\
\hline Estilo de Gestão & (Medeiros, et al., 2010, p. 4) & Servidores e Comunidade \\
\hline Condições de Trabalho & (Medeiros, et al., 2010, p. 7) & Servidores (ativos e desligados) \\
\hline Vínculo com a comunidade & (Medeiros, et al., 2010, p. 8) & Comunidade (usuários) \\
\hline Oportunidades de mercado & (Medeiros, et al., 2010, p. 7) & Servidores (ativos e desligados) \\
\hline
\end{tabular}

Fonte: Elaborado pelas autoras, com base na pesquisa realizada. 


\section{Resultados do Estudo}

\subsection{Análise do índice de turnover}

Pelos dados reportados na Tabela 1, por meio do cálculo, pode-se perceber uma variação significativa na média de turnover no período de 2016 a 2020. Evidencia-se que houve um aumento bastante expressivo na taxa de turnover no ano 2018 e, no ano 2019, configurou-se como a menor taxa de movimentação de pessoal.

Tabela 1 - Índice e média de turnover entre os anos 2016 e 2020.

\begin{tabular}{|l|c|c|c|c|c|c|}
\hline Ano & Meta & Turnover & $\begin{array}{c}\text { Média } \\
\text { Anual }\end{array}$ & $\begin{array}{c}\text { Número de } \\
\text { Funcionários }\end{array}$ & Admissão & Desligamento \\
\hline $\mathbf{2 0 1 6}$ & $5 \%$ & $15.00 \%$ & $15.00 \%$ & 20 & 2 \\
\hline $\mathbf{2 0 1 7}$ & $5 \%$ & $7.50 \%$ & $3.75 \%$ & 20 & 2 \\
\hline $\mathbf{2 0 1 8}$ & $5 \%$ & $35.00 \%$ & $11.67 \%$ & 20 & 6 \\
\hline $\mathbf{2 0 1 9}$ & $5 \%$ & $5.00 \%$ & $1.25 \%$ & 20 & 1 & 1 \\
\hline $\mathbf{2 0 2 0}$ & $5 \%$ & $17.50 \%$ & $3.50 \%$ & 20 & 4 & 3 \\
\hline
\end{tabular}

Fonte: Sistema de Informação da Atenção Básica (SIAB). Cadastro Nacional de Estabelecimentos de Saúde (CNES).

Os dados mostram que a taxa de turnover nas equipes em 2016 foi de 15\%, seguido por queda no ano seguinte para 7,5\%, e em 2018 teve a maior taxa do período com 35\%, praticamente o dobro de 2016. Em 2019 voltou a ter queda ficando em 5\%, período em que se manteve adequada, em 2020 subiu novamente, chegando a 17,5\%. Isso mostra que a taxa de turnover nas equipes da ESF do município é instável, podendo ser alta em determinado período, médio e baixo no período seguinte.

O alto índice de turnover em 2018 se explica porque, de acordo com os dados, nesse ano se desligaram das equipes da ESF três médicos generalistas, cada um de uma equipe, duas fisioterapeutas e uma enfermeira. No mesmo ano foram admitidos três médicos, duas enfermeiras, uma fisioterapeuta, uma nutricionista e um técnico de enfermagem. Toda essa movimentação de pessoal fez com que 2018 tivesse o maior índice de turnover do período.

Conforme o Gráfico 1, observamos que, dentre as categorias profissionais dessas equipes, as maiores movimentações são, em primeiro lugar, dos médicos generalistas com 33,3\% e, em segundo lugar, dos enfermeiros com $26,7 \%$.

Os resultados mostram que o turnover dos médicos e enfermeiros é superior ao restante das categorias profissionais, embora se mostre irregular de um ano para outro. Isso demonstra um dos maiores desafios enfrentados pelos municípios pequenos na implementação dos serviços de saúde da família, que é a dificuldade de contratação e fixação dos profissionais médicos e enfermeiros nas equipes. Isso pode acarretar prejuízos aos serviços de saúde do município, pois gera a quebra do vínculo e insatisfação da população. 
Gráfico 1. Saídas e admissões 2016 a 2020.

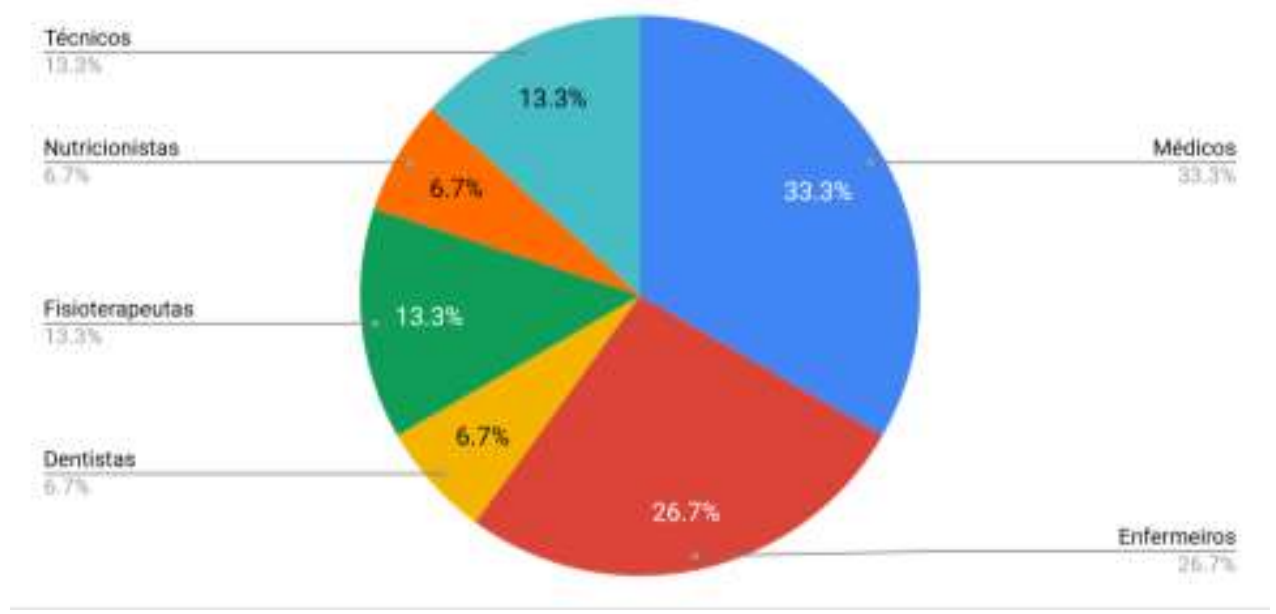

Fonte: Elaborado pelas autoras, com base na pesquisa realizada.

O estudo indica que a maioria dos contratos de trabalho dos profissionais das equipes da ESF no município de Rio Grande do Piauí é sem estabilidade, já que a forma de contrato por prazo determinado é de $75 \%$, bem maior que os estatutários que são apenas 25\%. Como pode-se observar no Gráfico 2.

Gráfico 2. Vínculo empregatício da ESF - 2016 a 2020.

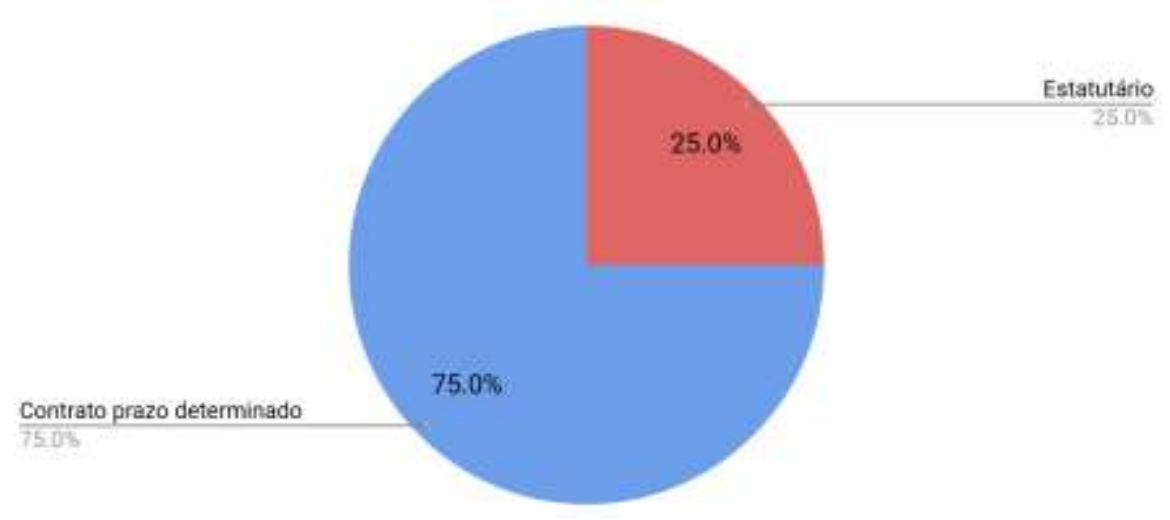

Fonte: Elaborado pelas autoras, com base na pesquisa realizada.

De acordo com os dados apurados na pesquisa, dentre os 14 (quatorze) servidores desligados no período em análise, apenas 4 (quatro) eram concursados e 10 (dez) eram contratados por prazo determinado. Fato que, com base nas teorias já abordadas, demonstra a importância da estabilidade desses profissionais para a permanência dos mesmos nas equipes e essa contratação provisória contribuiu para que o índice de turnover chegasse a nível tão alto em 2018. Visto que, ao término do contrato, os profissionais vão trabalhar em outros municípios e buscam outras oportunidades que lhes tragam a segurança que o concurso público pode proporcionar.

A estabilidade no trabalho é considerado um dos critérios para o sucesso da ESF em um município. Para que o profissional conheça a comunidade, sua história e assim, possa maximizar ações estratégicas de saúde. (Medeiros, et al., 2008) Porém, ainda que a estabilidade fortaleça o vínculo com a comunidade, a política da Atenção Básica não deixa claro a obrigatoriedade do concurso público, ficando a critério das prefeituras a forma de contratação (PNAB, 2012) Tonelli, et al. 
(2018) afirma que a ESF precisa avançar na gestão de pessoas no que se refere à contratação de pessoal, visto que, contratos temporários precarizam as relações de trabalho tornando as equipes incompletas e dificultando, assim, o cuidado com a população.

\subsection{Análise das entrevistas}

\section{Categoria 1: Papel do gestor}

Dentro desta categoria foi pesquisado como a gestão da Secretaria de Saúde do Município acompanha o índice de turnover dos profissionais da ESF. Constatou-se que não é realizada nenhuma ferramenta de cálculo para identificação desses dados. Como fala o entrevistado 01: "Não realizamos esse cálculo. Sabemos que existe a rotatividade, porém não utilizamos nenhuma ferramenta para identificar esses dados, até por conta que a equipe é pequena, então a percepção dessas demissões e admissões dos profissionais são notáveis facilmente."

Ainda dentro desta perspectiva buscou-se o entendimento sobre como é realizada a atração e fixação desses profissionais que prestam serviços à Atenção Básica. Segundo fala da entrevistada, são tomadas uma série de medidas na tentativa de favorecer e estimular esses profissionais, consequentemente incentivando-os na permanência de suas respectivas funções, como citado: "flexibilidade nos horários desses profissionais, assim como uma redução na carga horária, pagamos uma boa remuneração e em dia, disponibilizamos a ajuda de custos com moradia, refeições, com o apoio necessário” (E.01).

Nota-se que tais esforços da gestão desenvolvem um papel importante para a permanência desses talentos, conforme Cunha e Martins (2015, p. 94) “A retenção de talentos torna-se um fator primordial para não se perder a aplicação de capital feita para a capacitação do funcionário que dá resultado."

Ademais, essa série de iniciativas elencadas anteriormente, para a permanência desses servidores, são utilizadas como um atrativo para as contratações, uma vez que, encontrar profissionais especialistas para trabalhar na ESF, principalmente médicos, foi citado como uma das maiores dificuldades enfrentadas pela gestão, em razão destes profissionais residirem em outras cidades: "a principal dificuldade é quanto a contratação de profissionais como psicólogos, enfermeiro e principalmente profissionais médicos, pois essas são pessoas que na maioria, são de outros municípios” (E.01) Uma das causas principais do turnover, dentre esses profissionais, é o distanciamento entre a cidade onde trabalha e a família. Um enfermeiro desligado afirmou que essa logística foi um dos fatores de seu desligamento: "primordialmente foi o fato de não residir na mesma cidade que trabalhava, então analisando o custo-benefício ficou inviável" (D.01)

\section{Categoria 2: Estilo de gestão}

Nota-se, na fala dos entrevistados, a insatisfação quanto ao modelo de tomada de decisão, quando se trata do sistema de saúde, isto é, decisões da gestão que são tomadas de forma autocrática, quando na verdade deveriam ser em grupo. "É preciso também que as medidas que são tomadas em relação ao sistema de saúde, sejam de baixo para cima" (S. 02), haja vista, são esses profissionais que conhecem a realidade que o município enfrenta.

Sobre o estilo de gestão, o apoio do gestor gera proximidade e sinergia nos processos, o que resulta em profissionais entusiasmados e mais dedicados, com mais responsabilidade frente às dificuldades. (Medeiros, et al., 2008)

Ainda dentro desta categoria, buscou-se saber a opinião dos servidores e ex-servidores entrevistados, sobre o que seria preciso para que a Estratégia Saúde da Família do município atuasse de forma mais qualificada. Observa-se que há uma gama de iniciativas que podem ser tomadas, como por exemplo, o entrevistado S.01 citou: "A grande sugestão é a participação efetiva da população, por meio de questionamentos, acompanhamentos e principalmente cobrando não só do profissional, mas também do gestor para que venha haver um maior investimento na saúde.”

As outras sugestões foram: 
"É preciso ouvir os profissionais para que assim desenvolvam medidas de acordo com a realidade de cada município que evidentemente são distintas; Os gestores também precisam ouvir a população, por intermédio de fóruns, simpósios reuniões, também deve ser realizada uma busca ativa através dos profissionais que estão em contato direto com a comunidade..." (S.02)

"Que houvesse maior proximidade da gestão com as equipes e das equipes entre si,... a questão política acaba se tornando superior até as próprias necessidades dos profissionais, deixam de lutar para não entrarem em atritos, evitar conflitos internos com a gestão" (D.01)

"Não deixar faltar materiais básicos como o simples aparelho de verificar a pressão, uma espátula para realizar um exame preventivo nas mulheres, os medicamentos, outra sugestão a ser colocada é que os gestores realizem planejamentos para que não faltem insumos para a população, que saibam gerir os recursos que vem para o município, que tenham compromisso com a população" (D.02)

Por meio dos relatos, percebe-se que, para uma melhoria nas equipes ESF, é preciso que os agentes nelas inseridos ou que dela necessitam, se movam, isto é, a população precisa ser um agente ativo nessa relação, indo buscar, por meio de cobrança e acompanhamento, um sistema de atendimento melhor, assim como a ESF necessita de uma gestão participativa.

Entende-se, que é necessário que haja maior interação entre a gestão e as equipes para o planejamento das ações:

"Maior proximidade da gestão com as equipes e das equipes entre si, pois é visivel uma certa fragmentação entre as equipes.... Falta união entre as categorias para lutarem por melhorias trabalhistas, a questão política acaba se tornando superior até às próprias necessidades dos profissionais." (D.01)

Cabe destacar que a influência político-partidária, de certa forma, acaba prejudicando a dinâmica da ESF, uma vez que profissionais ficam retraídos frente às necessidades, para não se envolverem em conflitos com a gestão ou sofrerem "perseguições". Nesse aspecto, Medeiros, et al., (2008, p. 4) defendem que ação de gestor por meio de interferência política "pode provocar a redução no entusiasmo, descontentamento, insatisfação, além de frustrar expectativas, sendo um dos motivos para a rotatividade."

\section{Categoria 3: Condições de Trabalho}

$\mathrm{Na}$ pesquisa realizada, com os servidores em exercícios e desligados, foi constatado uma série de problemas enfrentados pelos profissionais no dia a dia, observou-se que o mais recorrente é a falta de medicamentos básicos, além de outros:

"Possui dificuldades de equipamentos, de medicações, de realização de exames, apesar de estar melhorando aos poucos, mas há uma necessidade ainda em relação a esses itens, Pois o profissional quer prestar um serviço de atendimento excelente, porem sempre falta alguma coisa" (S.01)

"Condições precárias de trabalho, falta de transporte para levar os profissionais para o atendimento nas comunidades da zona rural; também a falta de profissionais na equipe, deixando famílias descobertas de atendimento, sobrecarga de trabalho, falta de medicação na farmácia básica, como também a falta de material básico." (D.02) 
"Uma das grandes dificuldades é quanto à estrutura da saúde, uma vez que o governo, os gestores veem a saúde como gasto, enquanto deveriam olhar como investimento, até porque mesmo a população paga por meio dos impostos para se ter uma saúde de qualidade." (S.02)

"As dificuldades foram muitas: como, por exemplo, a de logística, de infraestrutura e mais especificamente naquele período foi o de remanejamento da agenda, assim como a má valorização de trabalho, falta de leis que garantam um plano de cargo de salários" (D.01)

Fica evidente a preocupação desses profissionais com a qualidade do serviço que estão prestando à população adscrita, pois tais dificuldades são consideradas como desfavoráveis para realização do atendimento de saúde com qualidade, haja vista que, por exemplo, a falta de transporte, impede principalmente a realização do atendimento às pessoas que residem na zona rural.

Desse modo, tais problemas acabam sendo fatores de insatisfação nos servidores por não conseguirem desempenhar seu papel de forma adequada, isso pode influenciar seu desligamento. "O profissional quer prestar um serviço de atendimento excelente, porém falta alguma coisa que acaba atrapalhando no desenvolvimento do trabalho." (S.01) Campos e Malik (2008, p. 7) sugerem que: "A melhoria do nível de satisfação dos profissionais é uma alternativa para o aumento da fixação dos mesmos."

\section{Categoria 4: Vínculo com a comunidade}

Nesta categoria buscou-se saber da população o seu olhar sobre a ESF da qual fazem parte. Nota-se um certo sentimento de satisfação por parte das famílias acompanhadas pelas equipes da zona urbana. conforme as falas:

"Na minha opinião, o atendimento da ESF tem sido satisfatório, pois sempre que nós precisamos de um atendimento em casa, para algum membro da família que se encontra impossibilitado, a equipe da ESF sempre se disponibiliza para fazer o atendimento domiciliar" (C.01).

"As visitas dos profissionais da ESF são realizadas mensalmente, sempre nos atualizando com informações e todas as vezes que ocorre de ter, por exemplo, vacina para minha bebê, logo eles nos avisam e com essa forma de tratamento nos sentimos bem cuidados por esses profissionais" (C.02).

Observou-se que as visitas mensais realizadas por esses profissionais são apontadas como um fator que, de certa forma, gera um vínculo com a comunidade, uma vez que sentem-se acompanhados e assistidos por essas equipes.

Entretanto, durante a pesquisa, constatou-se que as famílias que residem na zona rural possuem sentimento oposto, um sentimento de insatisfação e abandono quanto ao atendimento da ESF, pois relatam a ausência desses profissionais em suas comunidades: "Não considero um bom atendimento, pois moro na zona rural e a visita que recebo dessa equipe, é do agente de saúde que é a cada seis meses." (C.03) Diante disso, nota-se que o acompanhamento mensal é um fator crucial no atendimento às famílias assistidas pela equipe da ESF, pois, assim como pode gerar um vínculo com a comunidade, pode-se também ser o motivo da quebra deste.

Há notória discrepância entre a qualidade do atendimento das equipes da zona urbana e zona rural. Enquanto aquela relata certo cuidado, esta reclama de falta de profissionais e demora no atendimento: "Muita das vezes que procurei o atendimento no posto, não fui atendido, pois os médicos residem fora do município, aconteciam imprevistos e não avisaram com antecedência, os pacientes esperam várias horas para serem atendidos." (C.03) O entrevistado afirma que a ausência de 
médico no posto é consequência deste residir em outro município. Isso retoma a análise de que a distância do trabalho pode prejudicar o vínculo e ainda ser uma das causas do turnover desses médicos.

Ainda dentro desta perspectiva, buscou-se saber dos entrevistados o que seria preciso para melhorar o atendimento prestado pela ESF. As respostas foram:

"Fazer um monitoramento das pessoas em situação de vulnerabilidade socioeconômica para serem atendidas pela equipe ESF, pois todo cidadão ou cidadã tem direito a saúde gratuitamente e em seguida encaminhados a Assistência Social e promover palestras conscientizando a população sobre a importância do trabalho que a equipe ESF desenvolve" (C.01)

"Capacitação em profissionais sempre que possível do município, para que os recursos investidos nesse profissional sejam retribuídos com a permanência dele, assim como em cuidados com a população. Também as visitas realizadas pelos agentes de saúde na zona rural devem ser feitas com mais frequência," (C.03)

As sugestões da comunidade são muito importantes e devem ser ouvidas, visto que são essas pessoas que recebem os cuidados e entendem onde estão as maiores deficiências no processo de atendimento.

\section{Categoria 5: Oportunidades de mercado}

Outra influência para o turnover é o mercado de trabalho, relacionado à melhores ofertas de empregos e à qualificação das categorias profissionais. (Medeiros, et al., 2008)

Em se tratando do médico, Medeiros, et al., (2008, p. 7) afirma que "O mercado de trabalho, para os médicos, é amplo, principalmente em municípios do interior do estado" com isso, surge a dificuldade de fixar esse profissional em uma unidade de saúde de cidades pequenas, visto que, pode haver melhores oportunidades de mercado e a possibilidade de obter melhores rendimentos, através do trabalho em mais de uma instituição.

Essa oferta foi uma das soluções encontradas pelo gestor entrevistado para a fixação dos médicos no município:

"Dentre as medidas tomadas para solucionar as dificuldades, tentamos adequar o agendamento dos plantões, assim como a possibilidade desses profissionais fazerem plantões no hospital da cidade, até mesmo como uma forma de incentivo para permanecerem mais tempo, manter os salários em dia" (E.01)

Uma das entrevistadas aponta que a busca por melhores oportunidades de emprego e crescimento profissional ter motivado seu desligamento da equipe: "O que me levou a me desligar da ESF de Rio Grande, foi a minha aprovação para enfermeira no concurso federal" (D.02)

Esse posicionamento, em relação à carreira, também é percebido nas falas dos profissionais atualmente em exercício: "Em relação ao futuro tenho expectativas de crescer profissionalmente, e consequentemente melhorar a saúde da população e no momento não tenho pretensão de sair da ESF de Rio Grande." (S.01) "Meus planos para o futuro é me especializar, se houver condições de trabalho no Brasil para odontologia na ESF, digo isto porque a saúde pública no Brasil está em uma situação muito complicada." (S.02) Através desses relatos percebe-se que o sucesso na carreira e a qualificação profissional são indicadores para a retenção desses profissionais de saúde. Esse também é o entendimento de Agapito, Filho e Siqueira (2015, p. 8) "à medida que cresce a percepção de sucesso na carreira do profissional, crescem também os indicadores de bemestar no trabalho, e, dessa forma, a intenção dos profissionais de sair das empresas em que trabalham é reduzida." 


\section{Considerações Finais}

Atendendo ao objetivo geral deste estudo, descobriu-se, por meio dos estudos dos autores já citados, das entrevistas aplicadas aos sujeitos dessa pesquisa e da análise de cada categoria mencionada acima, que o turnover influencia negativamente o trabalho dos profissionais das equipes ESF ao gerar sobrecarga de trabalho advindo da falta de integrantes das equipes, restando ao pessoal ativo atender a demanda remanescente de pacientes insatisfeitos. Essa sobrecarga, aliada à falta de condições de trabalho, gera insatisfação nos trabalhadores e, por consequência, o aumento do turnover e a quebra do vínculo gerado entre os profissionais e a população.

Em resposta ao primeiro objetivo específico, conforme informações trazidas pelo cálculo, o turnover dos profissionais de saúde da ESF é considerado alto em determinados períodos, visto que o índice apresenta certa irregularidade de um período para o outro. Além disso, percebe-se que o turnover dos médicos e enfermeiros é superior ao do restante das categorias profissionais.

Outro fato importante, apontado pela análise dos dados, é que a grande maioria dos vínculos de trabalho dos profissionais da ESF no município é sem estabilidade, ou seja, contratos por prazo determinado. Os contratos temporários associados às relações trabalhistas precárias, resultam em problemas de fixação de pessoal, o que gera uma equipe desfalcada e dificuldades no atendimento à comunidade.

Em atenção ao segundo objetivo específico, por meio da entrevista, foi possível descobrir alguns fatores negativos que foram apontados como influência para o turnover. Através da análise de conteúdo, esses fatores foram apurados em cinco categorias de estudo: papel do gestor, estilo de gestão, condições de trabalho, vínculo com a comunidade e oportunidades de mercado. E as descobertas mais importantes foram:

Na categoria papel do gestor, é necessário frisar que a gestão não calcula o turnover dos servidores da ESF, dito isso, identificou-se problemas com os recursos financeiros da saúde, visto que há dependência desses recursos que vêm do Governo Federal. Essa dependência também atrapalha o desenvolvimento dos trabalhos das equipes.

Na categoria estilo de gestão, há insatisfação na relação do gestor com as equipes, pois estas apontam autoritarismo do gestor. As decisões não são em conjunto, ou seja, esse autoritarismo, somando-se a questões político-partidárias, é refletido nas equipes em forma de desunião e conflitos internos, causando insatisfação no trabalho.

$\mathrm{Na}$ categoria de condições de trabalho, percebe-se que a falta de medicação e equipamentos básicos causam uma insatisfação nos pacientes, consequentemente, isso atinge negativamente os profissionais que realizam o atendimento direto, $o$ que resulta em igual insatisfação desses profissionais, essa cadeia de insatisfação acaba motivando o turnover.

$\mathrm{Na}$ categoria vínculo com a comunidade, percebe-se que o atendimento das equipes da ESF na cidade tem sido satisfatório, porém observou-se que há uma falha no atendimento às famílias na zona rural, que, segundo os relatos, é por demora das visitas domiciliares, falta e atraso dos profissionais médicos, o que também é visto como uma situação que gera muitas reclamações, culminando assim na quebra de vínculo entre os profissionais e a comunidade.

$\mathrm{Na}$ categoria oportunidades de mercado, influencia o turnover quando o mercado de trabalho oferece melhores condições aos profissionais de saúde, especialmente ao médico, para o qual a oferta de emprego em pequenos municípios é ampla, o que favorece o trabalho em várias instituições. Outro fator é a procura por concursos públicos em instituições de nível mais elevado, na expectativa de crescimento profissional.

Esses problemas refletem a realidade complexa que os gestores e as equipes de saúde da família enfrentam no desempenho do trabalho.

E, para o terceiro e último objetivo específico, foi possível encontrar algumas alternativas para tentar solucionar esses problemas de forma que satisfaça os usuários e os profissionais de saúde, por meio das sugestões dos entrevistados e descobertas dos autores. São elas: contratação de profissionais de saúde residentes no município; melhor uso dos recursos 
públicos da saúde, não deixando faltar insumos, equipamentos, nem transporte para atendimento à população; capacitar os profissionais da ESF; união entre as categorias profissionais para lutarem por melhorias trabalhistas; evitar uso de influência político-partidária na gestão e liderança participativa, que melhor beneficia a permanência dos profissionais, visto que os resultados mostram que os gestores precisam ouvir os profissionais que estão em contato direto com o paciente. E a população precisa ter voz ativa para questionar, cobrar e acompanhar o uso dos recursos públicos.

Este estudo contribuiu para o conhecimento da realidade enfrentada pelos profissionais de saúde da família no desempenho de suas atividades, suas dificuldades e expectativas de trabalho, mostrou, pela ótica da comunidade, a satisfação e insatisfação com os serviços de saúde e a tentativa do gestor de buscar soluções para os problemas da saúde do município.

As limitações estão relacionadas ao curto prazo para organização dos sujeitos da pesquisa, realização da coleta de dados e posteriormente a análise do conteúdo. Outra limitação foi no universo da pesquisa, o estudo limitou-se ao município de Rio Grande do Piauí, assim, não sendo possível comparar com a realidade da ESF de outros municípios. Além disso, houve a impossibilidade de se atingir todos os servidores das Equipes Saúde da Família, restringindo-se a uma pequena parte deles. Ademais, outra limitação encontrada está relacionada à quantidade restrita de publicações sobre o estudo da rotatividade no setor público. Fato este, que restringiu o estudo bibliográfico desta pesquisa.

Para futuras pesquisas, sugere-se que sejam realizados mais estudos sobre a rotatividade no serviço público, com a aplicação de pesquisa em um público maior e com abrangência superior ao espaço de um único município, para que se tenha mais conhecimentos dos impactos que esta movimentação de profissionais pode causar ao setor público. É importante que as Estratégias Saúde da Família sejam estudadas com maior frequência, pois ainda há muitos desafios para superar, especialmente quanto à contratação dos profissionais médicos e enfermeiros em cidades pequenas.

\section{Referências}

Arantes, L. J., Shimizu, H. E., \& Hamann, E. M. (2016). Contribuições e desafios da Estratégia Saúde da Família na Atenção Primária à Saúde no Brasil: revisão da literatura. Programa de Pós-Graduação em Ciências da Saúde, Universidade de Brasília. Brasília DF.

Agapito, P. G., Filho, A. P., \& Siqueira, M. M. M. (2015). Bem-estar no trabalho e percepção de sucesso na carreira como antecedentes de intenção de rotatividade. RAM, Rev. Adm. Mackenzie, 16(6), 71-93.

Borges, N. S., Santos, A. S., \& Fischer, L. A. (2019). Estratégia de Saúde da Família: Impasses e desafios atuais. Saúde em Redes.

Bardin, L. (2004). Análise de Conteúdo (L'Analyse de Contenu): Presses Universitaires de France.

Campos, C. V. A. (2005). Por que o médico não fica? Satisfação no trabalho e rotatividade dos médicos do Programa de Saúde da Família do município de São Paulo: Escola de Administração de Empresas.

Campos, C. V. D. A., \& Malik, A. M. (2008). Satisfação no trabalho e rotatividade dos médicos do Programa de Saúde da Família: RAP.

Cunha, N. C., \& Martins, S. M. (2015). Retenção de talentos frente às mudanças no mercado de trabalho: uma pesquisa bibliográfica. Revista Getec, 4 (8), 90 109.

Departamento Intersindical de Estatística e Estudos Socioeconômicos - DIEESE. (2016). Rotatividade no mercado de trabalho brasileiro: 2012 a 2014. São Paulo, SP.

Gil, A. C. (2002). Como elaborar projetos de pesquisa: Atlas.

Instituto Brasileiro de Geografia e Estatatística - IBGE. Censo demográfico, 2010. (2020). https://www.ibge.gov.br/cidades-e-estados.

Korn Ferry 2019. (2020). The Global Talent Crunch. http://www.kornferry.com/future-of-work.

Longo, F. (2007). Mérito e flexibilidade: gestão das pessoas no setor público: Fundap.

Medeiros, C. R. G., Junqueira, A. G. W., Schwingel, G., Carreno, I., Jungles, L. A. P., \& Saldanha, O. M. F. L. (2010). A rotatividade de enfermeiros e médicos: um impasse na implementação da Estratégia de Saúde da Família. Ciência \& Saúde Coletiva.

Mota, M. M., Levy, E., \& Coelho, F. (2016). Fatores que Afetam a Rotatividade e Evasão de Talentos na Administração Pública Brasileira - O Caso dos Gestores Públicos. Conselho Nacional de Secretários de Estado da Administração - CONSAD.

Magnago, C., \& Pierantoni, C. R. (2015). Dificuldades e estratégias de enfrentamento referentes à gestão do trabalho na Estratégia Saúde da Família, na perspectiva dos gestores locais: a experiência dos municípios do Rio de Janeiro (RJ) e Duque de Caxias (RJ) Saúde Debate, 39(104), 9-17. 
Research, Society and Development, v. 10, n.5, e30310514744, 2021

(CC BY 4.0) | ISSN 2525-3409 | DOI: http://dx.doi.org/10.33448/rsd-v10i5.14744

Minayo, M. C. S. (2001). Pesquisa social: teoria, método e criatividade: Vozes.

Santos, R. C. A., \& Miranda, F. A. N. (2016). Importância do vínculo entre profissional-usuário na Estratégia Saúde da Família. Revista de Enfermagem/ UFSM, 6(3), 350-359.

Política Nacional de Atenção Básica - PNAB. (2012). Departamento de Atenção Básica: Ministério da Saúde.

Tonelli, B. Q., Leal, A. P. R., Tonelli, W. F. Q., Veloso, D. C. M. D., Gonçálves, D. P., \& Tonelli, S. Q., (2018). Rotatividade de profissionais da Estratégia Saúde da Família no município de Montes Claros, Minas Gerais, Brasil. Revista da Faculdade de Odontologia/UPF, Passo Fundo, 23(2), 180-185.

Tamayo, A., \& Paschoal, T. (2003). A relação da motivação para o trabalho com as metas do trabalhador. Revista de Administração Contemporânea, 7(4), 3354 .

Universidade Federal do Piauí. (2018). Projeto Pedagógico do Curso de Bacharelado em Administração à Distância: UFPI. 